\title{
A Theory of Wavelength Dependence in Ultrahigh Fre- quency Transhorizon Propagation Based on Meteorological Considerations ${ }^{1}$
}

\author{
Ralph Bolgiano, Jr. ${ }^{2}$
}

(October 26, 1959)

\begin{abstract}
Recent radio data indicate that the wavelength dependence of ultrahigh frequency transhorizon propagation varies widely in time. This is in contradiction with theoretical explanations previously set forth. Each attempt to account for the underlying effects of ever-present atmospheric motions has, in the past, pointed toward a unique form of the dependence. Extensive discussions have resulted as to the validity and relative merits of the various forms, but at no time has a variable wavelength dependence been proposed.

Since scatter propagation theory has predicted so satisfactorily the broad aspects of the radio signals, it is retained as the basis for further analysis. A new model is developed for the structure of refractive index fluctuations induced by turbulence. Grounded on a theory of homogeneous turbulence in a stably stratified atmosphere, which has been developed concurrently by the author, this new model provides an explanation for the observed distribution of wavelength dependence. It suggests that at times when the dynamic stability of the air within the scattering volume is neutral the received power should be nearly independent of radio wavelength. On the other hand, when the atmosphere is dynamically stable the signal strength should be proportional to the square, or higher power, of the wavelength.

These predictions have been tested by comparing the results of a scaled-frequency experiment with simultaneous meteorological data gathered along the path. Richardson's number for the 1- to 3-kilometer layer, within which the principal scattering volume lies, has keen employed as an index of dynamic stability, though it falls short of ideal in some respects. The 0.8 value of correlation found between Richardson's number and the wavelength dependence is highly suggestive that a relation of the nature predicted does, in fact, exist.
\end{abstract}

\section{Introduction}

A central prediction of the Booker-Gordon [1] $]^{3}$ scatter model for extra-diffraction tropospheric propagation was that the scatter loss should be independent of the radiofrequency. This matter of wavelength dependence has received considerable attention over the intervening years $[2,3,4,5,6,7]$. Various other explanations of the underlying electromagnetic phenomenon have been put forth, and several frequency scaling laws have been suggested. Those proposals that have met with greatest favor, both because of their relative simplicity as well as their ability to predict satisfactorily the broad aspects of nearly all the empirical data, are the single-scattering models such as Booker and Gordon [1] employed. Even among the proponents of these models there has been disagreement.

Perhaps this disagreement is most familiar when stated in terms of the spatial correlation of the deviations of refractive index, e.g.,

$$
R_{n}(\underset{\sim}{\xi}) \equiv \overline{\delta n(x, t) \delta n(\underset{\sim}{x}+\underset{\sim}{\xi}, t)} .
$$

It is, however, more readily interpreted when put in

1 This research was supported by the U.S. Air Force Cambridge Research Center under Contract No. AF 19(604)-3494, with Cornell University.

2 School of Electrical Engineering, Cornell Uni $\nabla$., Ithaca, N.Y. (invited paper).

3 Figures in brackets indicate the literature references at the end of this paper. terms of the mean square wavenumber spectrum of fluctuations of $n$ within the volume $V$,

$$
\begin{gathered}
\overline{\left|\delta A_{n}(\underset{\sim}{k}, t)\right|^{2}} . \\
\delta A_{n}(\underset{\sim}{k}, t) \equiv \int_{V} \delta n(\underset{\sim}{x}, t) \exp \{-\underset{\sim}{k} \cdot \underset{\sim}{x}\} d \underset{\sim}{x}, \\
\frac{\left.\delta A_{n}(\underset{\tilde{V}}{k}, t)\right|^{2}}{V}=\int_{\xi} R_{n}(\underset{\sim}{\xi}) \exp \{-\underset{\sim}{k} \cdot \underset{\sim}{\xi}\} d \xi,
\end{gathered}
$$

the latter integration being over all of $\underset{\sim}{\xi}$-space. This ease of interpretation comes about because the scattering coefficient $\sigma$ may be expressed directly in terms of this spectrum [3].

$$
\sigma=\frac{4 \pi^{2} \sin ^{2} \chi}{\lambda^{4}} \frac{{\overline{\delta A_{n}\left(k_{\sigma}, t\right)}}_{\tilde{V}}^{2}}{V} .
$$

The wavenumber $k_{\sigma}$, at which the spectrum must be evaluated, is determined by the radio wavelength and the path geometry. Its magnitude is given by

$$
k_{\sigma}=\frac{4 \pi}{\lambda} \sin \frac{\beta}{2}
$$

in which $\beta$ is the scatter angle. If the deviations of refractive index are isotropic, at least at scales of 
order corresponding to $k_{\sigma}$, the mean square fluctuation spectrum is a function of wavenumber magnitude only. Then the scattering coefficient may be expressed as

$$
\sigma=\frac{8 \pi^{4} \sin ^{2} \chi}{\lambda^{4}} \frac{E_{n}\left(k_{\sigma}\right)}{k_{\sigma}^{2}}
$$

in which

$$
\left.E_{n}(k)=\frac{k^{2}}{8 \pi^{3} V} \int_{4 \pi} \overline{\left.\delta A_{n}(\underset{\sim}{k}, t)\right|^{2}} d \Omega \underset{\sim}{k}\right),
$$

the integral extending over all directions of $k$. Thus, the wavelength and angle dependence of the scattering coefficient and, indirectly, the wavelength dependence of the received power stem from the form of this spectrum.

Conversely, to the extent that the single-scattering approximation is valid, the structure of the refractive index variations may be studied experimentally via radio data. This latter approach led Norton [8] to propose his log-normally modified $\rho K_{1}(\rho)$ correlation function for the $n$-fluctuations. The $\rho K_{1}(\rho)$ form had been suggested previously [9] since it leads to a $\lambda$ to the first power dependence in $\sigma$, in agreement with the mean of radio data collected on numerous paths over several years [5]. Villars and Weisskopf [4] suggested a model of turbulent mixing which vields the corresponding spectral form. When their work first appeared, the justification it seemed to afford the empirical results led to its rapid acceptance. More recently, however, an inconsistency has been pointed out in this model of the structure of fluctuation spectra [10]. Wheelon [11] has discussed the matter at some length, but his arguments have been questioned further by Bolgiano [12] and the uncertainty regarding the Villars-Weisskopf mixing theory remains.

Applying the statistical theory of isotropic turbulence, Batchelor [13] has shown that $\sigma$ should exhibit a $\lambda^{-1 / 3}$ wavelength dependence. This is a consequence of the $k^{-5 / 3}$ wavenumber dependence of the mean square refractive index fluctuation spectrum shown in figure 1 . This $k$-dependence is, in turn, the

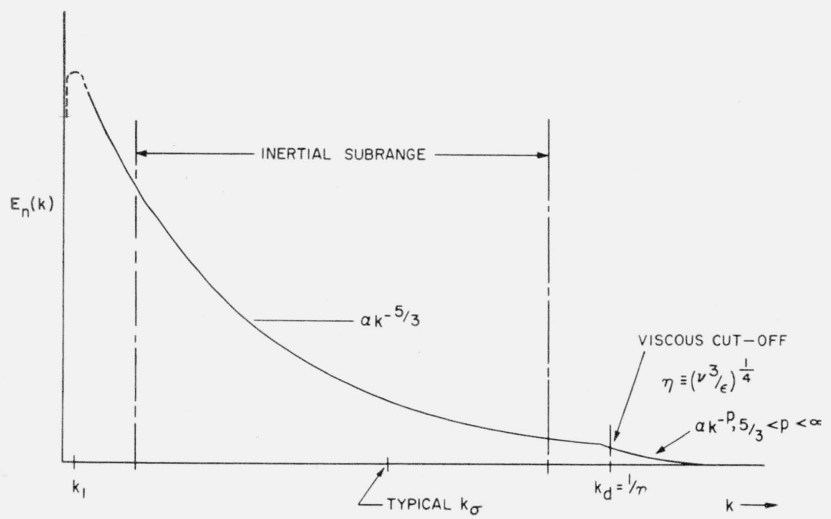

FIGURE 1. Spectrum of mean square refractive index fluctuations.

Neutral atmosphere-isotropic turbulence. universal form [14] characteristic of the inertial subrange, that interval lying between the anisotropic mixing eddies at the large scale end of the spectrum (small $k$ ) and the very small size components, subject to severe molecular "dissipation," at the other extreme. In the isotropic theory this dissipation, or viscous, cutoff of the spectrum occurs at a scale $\eta$ given by

$$
\eta=\left(\frac{\nu^{3}}{\epsilon}\right)^{1 / 4}
$$

where $\nu$ is the kinematic viscosity of the air and $\epsilon$ is the rate (per unit mass of the fluid) at which turbulent energy is converted into heat by the action of viscosity. On the basis of previous estimates of $\epsilon$ that have been made by the meteorologists, $\eta$ is of the order of millimeters, whereas the scales involved in radio scattering (corresponding to $k_{\sigma}$ ) are typically of the order of meters. Turbulence theory, therefore, predicting a $\lambda^{-1 / 3}$ dependence, would appear to be in disagreement with the bulk of the radio data for which the mean form is $\lambda^{1}$.

Partly, no doubt, because of this inability of scatter theory, as based on turbulence-induced dielectric inhomogeneities, to agree with the empirical results, but also because there is strong evidence of refractive index stratification in the lower troposphere, Bauer [15] and Friis, Crawford, and Hogg [7] have proposed coherent partial reflections from layers to explain transhorizon propagation. By judicious choice of the size of the layers (relative to Fresnel zones) and of the vertical thickness of the index gradient modification regions, the latter authors have arrived at a wavelength dependence for the received power of $\lambda^{1}$. It is interesting to note that by other choices (perhaps less sound physically) agreement can be achieved with any wavelength dependence in the range $\lambda^{-2}$ to $\lambda^{4}$.

In order to account for the signal levels observed, the angular distribution of received power, and the fading character of the signals, these same authors have found it necessary to assume large numbers of layers, randomly located and moving. Viewed in this light, it would seem this reflection model is simply another approach to scattering by turbulent anisotropic irregularities. If so, the appropriate wavelength dependence will stem from the refractive index correlation and spectrum functions. These functions, in turn, should have some sound basis in dynamic meteorology, including atmospheric turbulence, which is seldom, if ever, entirely absent.

\section{Distribution of the Wavelength Dependence}

The lack of agreement between the predictions of turbulence theory and the propagation data is even more striking when the results of a scaled-frequency experiment [16] are considered. In that experiment, two channels, one at $417 \mathrm{Mc}$, the other at 2,290 Mc, were operated simultaneously over the same path using scaled antennas. Thus, the volume common 
to the transmitting and receiving beams was identical on the two frequencies and any differences in observed transmission loss can be identified directly with the wavelength dependence of $\sigma$. From the data of figure 2 , it is apparent that no one "uni-

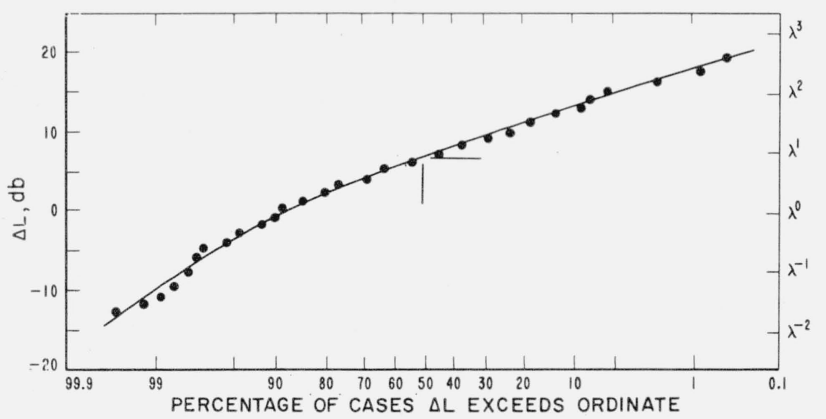

FIGURE 2. Distribution of wavelength dependence.

Corrected differences in measured hourly median basic transmission loss, $\Delta L$ for scaled-frequency experiment: 417.05 Mc versus 2,290 Mc, Round HillCrawford's Hill, February 11, 1957 to July 11, 1957, 241 cases. (After ChisCraw ford's Hill, February 11,
holm, Roche, and Jones [16].)

versal" spectral form holds, since the wavelength dependence, as indicated by the corrected difference in the observed values of hourly median basic transmission loss on the two channels $(\Delta L)$, varies widely. ${ }^{4}$ True, the median of the distribution corresponds roughly to $\lambda^{1}$. The variation, however, ranges from $\lambda^{-3 / 2}$ to $\lambda^{5 / 2}$, i.e., from $k^{-1 / 2}$ to $k^{-9 / 2}$ for the fluctuation spectrum. This then must disqualify any theoretical explanation in terms of a model which predicts a unique wavenumber dependence. Yet turbulence theory, as it has been applied to the problem, has led to such a result.

This seeming dilemma could be overcome if only the viscous cutoff scale $\eta$ lay in the meter range much of the time, rather than in the millimeter range. Consideration of figure 1 will aid in visualizing this point. If $k_{d}$ were to fall at or below $k_{\sigma}$, the wavenumber dependence of the spectrum in the vicinity of $k_{\sigma}$ would be "stronger" than the $k^{-5 / 3}$ form of the inertial subrange. Wide variation of $\eta$ in time could then account for the distribution of wavelength dependence of the received power which has been observed.

These large values of $\eta$, however, could occur only in conjunction with exceedingly small dissipation rates; and very small $\epsilon$, in turn, requires either a correspondingly low turbulent intensity or a more rapid decrease of kinetic energy density with increasing wavenumber than the universal equilibrium theory [18] indicates. This latter solution implies the progressive removal of kinetic energy from the spectrum by some mechanism other than viscosity.

Very low turbulent intensity, which may result when a given field of turbulence has decayed appreciably, is certainly a prerequisite to the existence of well-formed layers of the type essential to Bauer's [15] coherent reflection model. When such circum-

\footnotetext{
${ }_{4}^{4}$ There is the question as to the statistical significance of this variation. This matter has been considered, and it has been found that the standard deviation of the empirical data is an order of magnitude larger than would be expected on the basis of statistical fluctuations alone [17].
}

stances prevail, an explanation based instead on turbulence theory (modified as above) has great merit since it does not require a complete absence of random air motions. It states only that such motions must be very "weak." Even this limited requirement, though it may be satisfied occasionally (in a strong inversion for example), is not the typical situation at the $5,000-\mathrm{ft}$ level. This conclusion is borne out by the meteorological evidence as to the usual intensity of turbulent velocities at this altitude [19]. It is several orders of magnitude higher than that of the low level turbulence needed if this explanation is to be applicable.

\section{Turbulence in Stably Stratified Media}

It should be noted, on the other hand, that the meteorological estimates of $\epsilon$ invariably have been made on the basis of some large-scale turbulence effect, such as the modification of the wind profile by eddy viscosity, together with the assumption that the dissipation rate is determined solely by this same large-scale turbulence. This is equivalent to assuming that the rate at which kinetic energy is transferred "up the spectrum" inertially, from larger to smaller eddies, is constant, independent of eddy size, until actual dissipation begins to take effect in the vicinity of the viscous cutoff. That is to say, no mechanism other than viscosity operates to remove kinetic energy from the turbulent hierarchy.

This approach does not adequately account for the effects of buoyancy forces which come into play in a stable atmosphere as a result of the less than adiabatic temperature lapse rate. A particle of air on moving vertically is no longer in static equilibrium with its surroundings. Continuing to travel up or down it works against the force of buoyancy and, as a consequence, has at least part of its (turbulent) kinetic energy converted to potential energy. Vertical flux of heat effected by eddy diffusion is a closely associated process and a familiar meteorological phenomenon.

An important result, therefore, of a stable density gradient is to provide the means by which kinetic energy may be extracted from the velocity field at various intermediate scales, thus voiding the assumption on which the estimates of $\epsilon$ depend. It is conceivable that for stable conditions these estimates have been high, perhaps by many orders of magnitude.

In a neutral atmosphere ${ }^{5}$ as in a uniform fluid, there being no preferred direction, it is not suprising that the smaller scale motions have a strong tendency toward isotropy. On the contrary, a stable temperature (and density) gradient must single out the vertical direction as unique. Consequently, an anisotropic, axisymmetric structure will be favored.

Under such circumstances the energy spectrum tensor takes the general form

${ }^{5}$ An atmosphere in which the mean potential temperature $\bar{\theta}$ is independent of height. 


$$
\left.\frac{1}{2} \Phi_{i i} \underset{\sim}{k}\right)=-k^{2} A(k)+\frac{1}{2} B\left(k^{2}, k_{z}^{2}\right)\left(1+\frac{k_{z}{ }^{2}}{k^{2}}\right),
$$

in which $A$ and $B$ are arbitrary scalar functions, and the total energy density may be written

$E(k)=-4 \pi k^{4} A(k)+\frac{k^{2}}{2} \int_{4 \pi} B\left(k^{2}, k_{z}^{2}\right)\left(1+\frac{k_{z}^{2}}{k^{2}}\right) d \Omega(\underset{\sim}{k})$.

Moreover, the spectral covariance between vertical velocity and density fluctuations $\Phi_{z \tilde{\omega}}(k)$, which is identically zero in an isotropic field, is of the form

$$
\Phi_{z \tilde{\omega}} \underset{\sim}{(k)}=F\left(k^{2}, k_{z}{ }^{2}\right)\left(1-\frac{k_{z}{ }^{2}}{k^{2}}\right)
$$

Since this velocity-density covariance plays an essential role in any interaction of the various eddies with the density gradient it seems likely that such interaction does occur, thereby tending to preserve the anisotropic structure. On the hypothesis that a state of equilibrium of this nature exists among those motions not too large in scale, it is possible to construct a theory of homogeneous turbulence in stably stratified media [20] much like the now familiar universal equilibrium theory for uniform fluids. Use is made of the methods of dimensional analysis, the determining parameters being

$\epsilon=$ the kinetic energy dissipation rate,

$\nu=$ the kinematic viscosity,

$\mathscr{D}=$ the molecular diffusivity,

$g / \hat{\rho}_{a}=$ the ratio of acceleration due to gravity to the reference potential density,

$\epsilon_{n}=$ the rate of "dissipation" of mean square refractive index fluctuations,

$\epsilon_{\tilde{\omega}}^{\tilde{}}=$ the rate of "dissipation" of mean square potential density fluctuations.

A prominent feature of this new model is an equilibrium range which, in general, ${ }^{6}$ divides into three parts: The buoyancy subrange, the inertial subrange, and the dissipation subrange. The buoyancy subrange is that portion at the large scale end in which buoyancy forces play a dominant role and the structure is anisotropic. It is within this interval that kinetic energy is extracted by conversion to potential energy. The rate at which kinetic energy is transferred inertially "up the spectrum" is here many times the dissipation rate $\epsilon$. As a consequence, the structure depends upon only $\epsilon_{\tilde{\omega}},\left(g / \hat{\rho}_{a}\right)$, and $\epsilon_{n}$. The energy and refractive index fluctuation spectra are given by

$$
\begin{gathered}
E(k) \sim \epsilon_{\tilde{\omega}^{2 / 5}}\left(g / \hat{\rho}_{a}\right)^{4 / 5} k^{-11 / 5}, \\
E_{n}(k) \sim \epsilon_{n} \epsilon_{\tilde{\omega}^{-1 / 5}}\left(g / \hat{\rho}_{a}\right)^{-2 / 5} k^{-7 / 5} .
\end{gathered}
$$

The inertial subrange lies in the middle of the equilibrium range, is dominated solely by the inertial transfer processes, and is isotropic. At these scales the anisotropy of the buoyancy subrange has been erased largely through the nondirectional effects of the pressure fluctuations. The spectral forms are those of the constant density theory, viz,

$$
\begin{gathered}
E(k) \sim \epsilon^{2 / 3} k^{-5 / 3}, \\
E_{n}(k) \sim \epsilon_{n} \epsilon^{-1 / 3} k^{-5 / 3} .
\end{gathered}
$$

The dissipation subrange contains the smallest scales (highest wavenumber components). It is in this region that the great bulk of viscous (molecular) dissipation occurs.

Figure 3 indicates the nature of $E_{n}$ in a typical

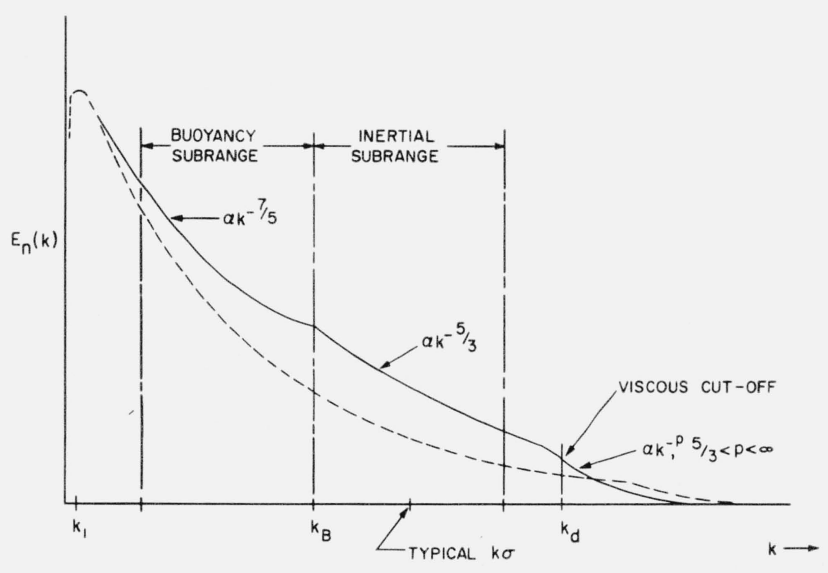

Figure 3. Spectrum of mean square refractive index fluctuations.

Stably stratified atmosphere-anisotropic turbulence.

case. The dashed curve shows the spectrum for a neutral atmosphere. The effect on the location of the viscous cutoff at $k_{d}$ should be noted in particular.

The buoyancy and inertial subranges are separated by a wavenumber $k_{B}$ given by

$$
k_{B} \equiv \frac{\epsilon_{\omega}^{3 / 4}\left(g / \hat{\rho}_{a}\right)^{3 / 2}}{\epsilon^{5 / 4}} .
$$

$\epsilon_{\tilde{\omega}}^{\tilde{}}$, being the rate at which mean square potential density fluctuations are dissipated by molecular diffusion, is also the rate at which the same quantity is generated through mixing of the mean potential density gradient by the large scale motions. Its value is therefore determined by the static stability of the atmosphere and the intensity of the primary mixing motions. On the other hand, $\epsilon$ depends not only upon the large scale velocity components but also upon the total rate at which kinetic energy is extracted from the turbulence in the buoyancy subrange. Thus, in a neutral or near neutral atmosphere $k_{B}$ is very small (large size eddy). There is then little or no buoyancy subrange; $\epsilon$ is determined by the large scale motions alone, and the universal equilibrium theory results eq (11) and (12) hold. In a highly stable situation, however, especially if there is relatively little mechanical excita- 
tion, ${ }^{7} k_{B}$ is very large and the inertial subrange may be entirely suppressed. Under these circumstances, $\epsilon$ being exceedingly small (even though the "turbulent intensity" observed at the large scales is moderate), the viscous cutoff may occur at eddies of the order of a meter in size.

The exact form that the spectra take in the dissipation range, beyond the viscous cutoff, is an unsettled matter. Batchelor [21] and Batchelor, Howells, and Townsend [22] have shed some light upon the limiting cases, $\mathscr{D}\langle\langle\nu$ and $\mathscr{D}\rangle\rangle \nu$. The case of primary interest for the troposphere $(\mathscr{D} \sim \nu)$ has yet to be treated satisfactorily.

Before leaving this subject it is interesting to note that there is some evidence, quite independent of the radio propagation phenomenon, which favors this stably stratified model. One of the predictions it yields is that the dispersion of a cloud of particles (a smoke puff, for example) is characterized by a phase of its development in which the diffusion rate increases as the fourth power of time. This compares with a second power form on the basis of the universal equilibrium theory and a constant rate for molecular diffusion. Analysis of a series of smoke puffs in the upper troposphere and lower stratosphere has shown that under conditions of near neutral stability and/or high wind and shear the diffusion rate is closely proportional to $t^{2}$ [23], while under conditions of high stability with light wind and weak shear it is more nearly proportional to $t^{4}$ (Bolgiano, unpublished). The available data are too few to be conclusive. Nonetheless, such evidence is certainly not contradictory.

\section{Relation of Wavelength Dependence to Dynamic Stability}

In an attempt to understand more fully the distribution of wavelength dependence, the propagation data of Chisholm, Roche, and Jones [16] and, when available, simultaneous meteorological data for the vicinity of the path have been scrutinized and compared [24]. For example, the winter (February and March) and summer (June and July) data have been plotted separately in figure 4 . Note that the stand-

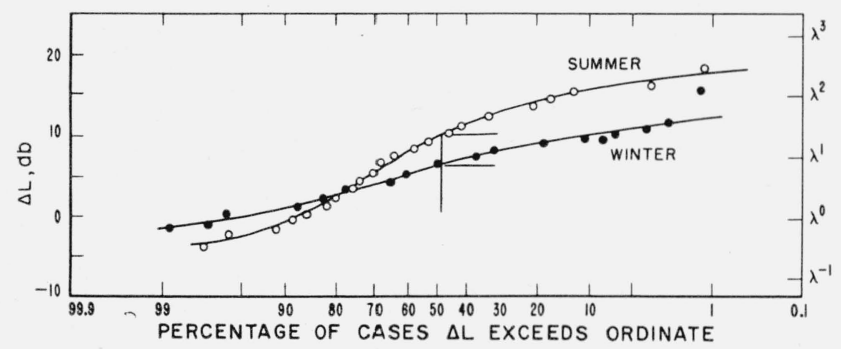

Figure 4. Seasonal variation in distribution of wavelength dependence.

Corrected differences in measured hourly median basic transmission loss, $\Delta L$, for scaled-frequency experiment: 417.05 Me versus 2,290 Mc, Round Hillfor scaled-frequency experiment: 417.05 Me versus 2,290 Mc, Round Hill-
Crawford's Hill. Winter: February and March 1957, 76 cases. Summer: June and July 1957, 80 cases.

7 Light winds and little or no shear. ard deviation is larger and the median value higher in summer than in winter. Though the variation from season to season appears distinct, there is no immediately obvious explanation. A similar conclusion may be reached regarding the diurnal cycle. The data have been replotted on a diurnal basis in figure 5. There is no pronounced diurnal variation in winter and even in summer only the lower limit shows a fairly well developed cycle.

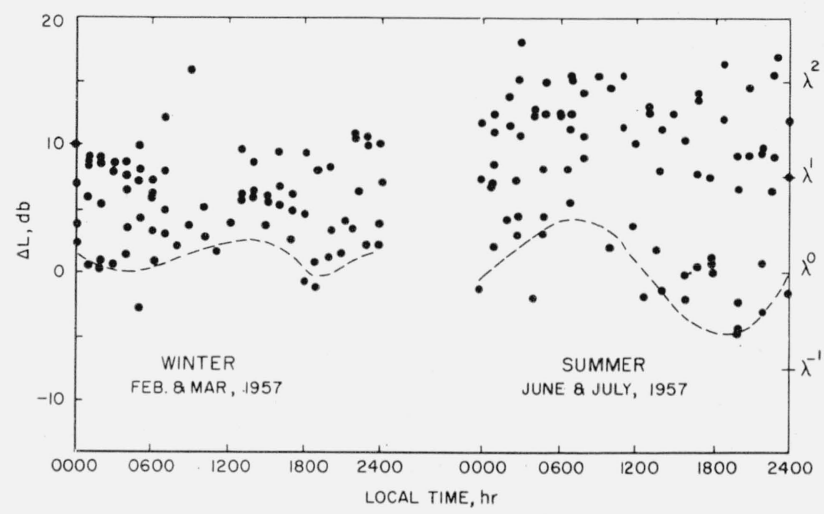

Figure 5. Diurnal variation of wavelength dependence. Corrected differences in measured hourly median basic transmission loss, $\Lambda L$
for scaled-frequency experiment: 417.05 Mc versus 2,290 Mc, Round HillCrawford's Hill.

Another prominent feature of the data is the coincidence of abrupt negative changes in the differential transmission loss $\Delta L$, with the occurrence of precipitation along the path. This is particularly noticeable when the radio data are "continuous" for several days and local or frontal showers cross the path. Finally, correlation between the wavelength dependence and the static stability of the atmosphere $(d \bar{\theta} / d z)$ has been found significant at the 0.05 level.

This gradient of mean potential temperature $\bar{\theta}$ is indicative of the strength of the buoyancy forces, which operate to oppose vertical motions when the gradient is positive, to accelerate them when it is negative. Its value depends upon the actual temperature gradient and upon the adiabatic temperature lapse rate of the atmosphere. This latter quantity, in turn, changes abruptly as the air becomes saturated, the change being such that less stable conditions result. The precipitation effect noted above could be accounted for on this basis. At least it is now possible to explain the above coincidence in terms of the observed correlation with static stability.

This correlation, as well as the seasonal and diurnal variation depicted in figures 4 and 5 , seem to be in qualitative agreement with the notions developed in sections $B$ and $C$. In summer when mean winds in the Long Island area ${ }^{8}$ are "light-to-variable" (though there are occasional intense thunderstorms) the median value of $\Delta L$ is high and the spread is

8 The Round Hill-Crawford's Hill path, on which the radio data were gathered is such that the major seattering volume is over Long Island and Long Island Sound. 
wide. In winter, on the contrary, when the mean winds are more uniformly intense the median power ratio is lower and the spread is narrower. Again in winter when there is relatively little consistent diurnal variation of weather (mean winds, temperature gradients, etc.) there is no pronounced daily cycle. In summer, however, when there is sometimes marked variation of wind, convection, and turbulence, from calm or light in the early morning hours to moderate or strong in the afternoon, one finds a fairly evident diurnal cycle.

Though such qualitative agreement with the proposed model is encouraging, it is desirable to test the predictions which can be made on the basis of that model in some more quantitative manner. Explicitly these predictions are: (1) That at times when mechanical excitation in the atmosphere (large scale horizontal motions, shear, etc.) dominates stability suppression, the wavelength dependence should favor a $\lambda^{-1 / 3}$ to $\lambda^{-3 / 5}$ form; and (2) at times of high static stability, however, and when winds are light, the wavelength dependence should tend toward $\lambda^{1}$ and $\lambda^{2}$ and higher powers.

The desired test might be achieved by investigating the correlation, if any, between an appropriate index of dynamic stability and the differential transmission loss, $\Delta L$. The choice, or formation, of a suitable index which can be evaluated readily from the generally available meteorological data presents some difficulty. Richardson's number $R i^{9}$ meets the specifications only approximately, at best. It fails to account properly for the turbulence-driving: capabilities of very large scale horizontal motions.

Nonetheless, $R i$ has been evaluated from radiosonde and rawinsonde data gathered in the vicinity of the path for the layer 1 to $3 \mathrm{~km}$ which includes the primary scattering volume. Mean winds at each level have been calculated and the mechanical excitation taken as the square of the total variation of mean wind over the chosen height interval. The resulting index values have been compared with $\Delta L$ for a number of "consistent" cases. These are instances for which the value of $\Delta L$ remained reasonably constant (within several decibels) for a minimum of $8 \mathrm{hr}$, although the transmission loss on the individual channels varied sometimes by 10 to $15 \mathrm{db}$. The data are presented in table 1.

The scatter plot in figure 6 indicates that the correlation is very pronounced. The value of sample correlation computed is 0.80 which, for this 20-point sample, establishes the lower (one-sided) 95-percent confidence limit, for the true correlation, at 0.6. That is to say, if the actual correlation is less than 0.6 , the sample correlation should be less than the observed value with a probability of 0.95 , or greater.

${ }^{\theta} \mathrm{Ri}$ is the ratio of the rate at which kinetic energy is extracted from the turbulence, by the effect of buoyancy forces, to the rate at which kinetic energy is transferred to the turbulence from the mean flow through interaction with the transferred to the turical wind shear.

TABLE 1. Data and computed results for "consistent" cases

Round Hill-Crawford's Hill Radio Link, February-July 1957. Meteorological data from, "daily series, synoptic weather maps, part II: northern hemisphere data tabulations",

\begin{tabular}{|c|c|c|c|c|c|c|c|}
\hline Case & $\Delta L$ & Precipitation & $\overline{(d T / d z)_{1-3}}$ & $\bar{T}_{1-3}$ & $d T / d z \mid$ adiab. & $|\Delta U|_{1-3}$ & $(R i)_{1-3}$ \\
\hline Date and time & $d b$ & & ${ }^{\circ} \mathrm{C} / \mathrm{km}$ & ${ }^{\circ} K$ & ${ }^{\circ} \mathrm{C} / \mathrm{km}$ & $\mathrm{m} / \mathrm{s}$ & \\
\hline${ }^{(2 / 28 / 1500-3 / 01 / 0500) \ldots . . . .}$ & 1 & $\Delta \therefore$ & -2.9 & 273 & -6.0 & 18. 0 & 1.4 \\
\hline $\begin{array}{l}(3 / 05 / 2200-3 / 06 / 0700) \\
\text { III }\end{array}$ & 9 & & -3.0 & 268 & -9.9 & 9.0 & 13.0 \\
\hline $\begin{array}{l}(3 / 18 / 1800-3 / 19 / 0600) \\
\text { IV }\end{array}$ & 3 & $T$. & -3.8 & 271 & -9.5 & 21.0 & 2.1 \\
\hline$\underset{\mathrm{V}}{(5 / 06 / 2000-5 / 07 / 0400) \ldots . . .}$ & -11 & & -9.5 & 272 & -9.9 & 9. 5 & 0.7 \\
\hline$(5 / 07 / 0600-5 / 07 / 1800) \ldots$ & 2 & & -6.8 & 278 & -9.9 & 13.5 & 2.4 \\
\hline $\begin{array}{c}\mathrm{VI} \\
(5 / 13 / 1200-5 / 13 / 2200) \\
\mathrm{VII}\end{array}$ & 6 & & -6.0 & 283 & -9.9 & 15. 0 & 2.5 \\
\hline $\begin{array}{l}(5 / 14 / 0000-5 / 14 / 1400) \\
\text { VIII }\end{array}$ & 9 & & -5.3 & 282 & -9.9 & 17. 5 & 2.1 \\
\hline$(5 / 21 / 1800-5 / 22 / 0400) \ldots$ & 6 & & -2.4 & 277 & -9.9 & 11.0 & 9.0 \\
\hline $\begin{array}{c}(5 / 22 / 0800-5 / 22 / 1800) \\
\quad \mathrm{X}\end{array}$ & 6 & & -1.1 & 282 & -9.9 & 13. 0 & 7.4 \\
\hline$(5 / 23 / 0000-5 / 23 / 0800)$ & 5 & $T_{-}$ & -3.6 & 284 & -9.5 & 10.5 & 7.9 \\
\hline $\begin{array}{c}\mathrm{XI} \\
(6 / 11 / 0100-6 / 11 / 1200)\end{array}$ & 14 & & -4.7 & 283 & -9.9 & 9.0 & 9.0 \\
\hline (6/11/1200-6/11/2300) & 15 & & -5.2 & 285 & -9.9 & 5. 5 & 21.2 \\
\hline$(6 / 24 / 1300-6 / 24 / 2200)$ & -1 & B $\infty$. & -6.3 & 286 & -7.3 & 8.0 & 2.2 \\
\hline (6/26/0700-6/26/1500) & 14 & & -5.2 & 285 & -9.9 & 7.5 & 12.1 \\
\hline$(6 / 26 / 2000-6 / 27 / 0800) \ldots$ & 11 & & -5.6 & 285 & -9.9 & 7.5 & 10.7 \\
\hline$\underset{(7 / 02 / 1300-7 / 03 / 0000)}{\mathrm{XVI}}$ & -2 & & -7.5 & 280 & -9.9 & 10.5 & 3.1 \\
\hline$\underset{(2 / 11 / 2200-2 / 12 / 0600)}{X V I I}$ & 8 & & -2.6 & 263 & -9.9 & 12. 0 & 7.8 \\
\hline (5/15/0600-5/15/1800) & 7 & $T$. & -6.3 & 283 & $-9 . \check{\delta}$ & 10.5 & 3.9 \\
\hline$(7 / 08 / 1600-7 / 09 / 0000)$ & 15 & & -7.5 & 284 & -9.9 & 8.5 & 4.6 \\
\hline$(7 / 10 / 1600-7 / 11 / 0600)$ & 10 & & -5.5 & 278 & -9.9 & 10.5 & 5.7 \\
\hline
\end{tabular}




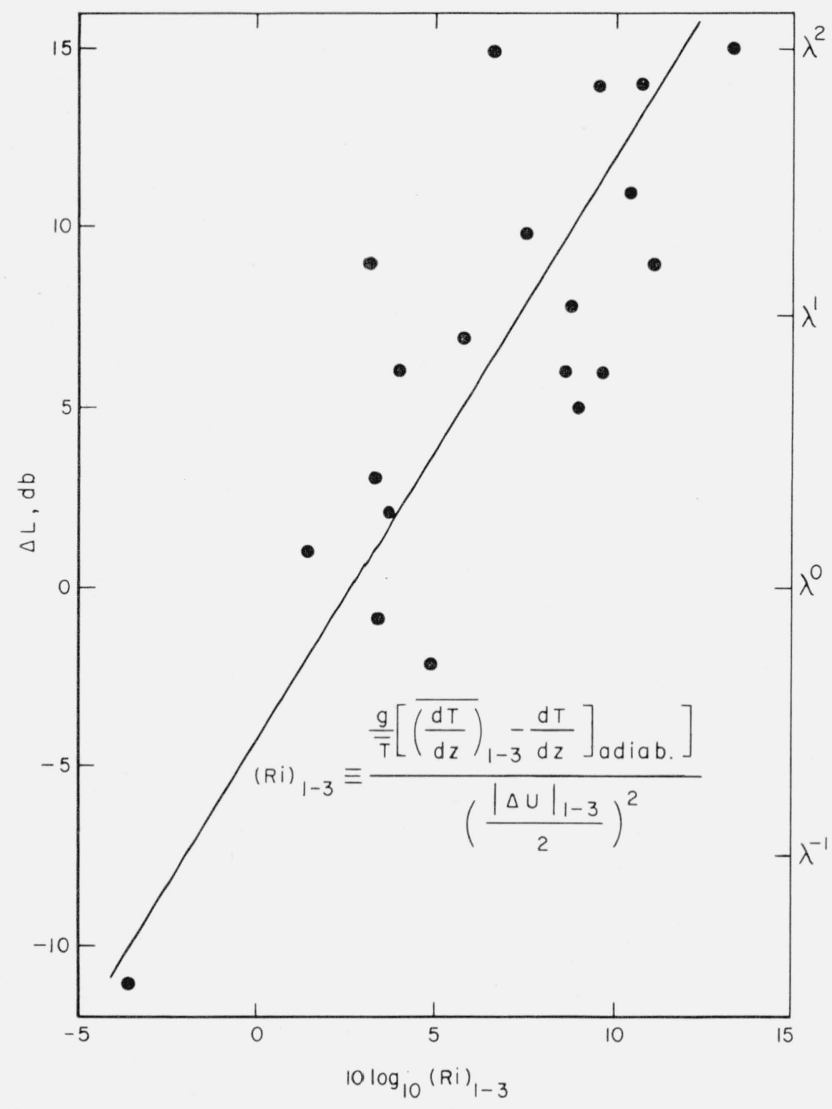

FIGURE 6. Variation of wavelength dependence with dynamic stability of the atmosphere in the scattering volume.

Sample correlation $r=0.80$.

\section{Conclusions}

A rather close relationship appears to exist between the wavelength dependence of transhorizon tropospheric propagation and the dynamic stability of the atmosphere in the region of space common to the transmitting and receiving antenna beams. Considered in terms of scatter theory this implies an association between the functional form of the spectrum of mean square refractive index fluctuations and dynamic stability. This in turn, when viewed in the light of statistical turbulence theory, seems to indicate a strong connection between the viscous cutoff scale and this meteorological index. Finally then, there appears to be in the radio data confirmation of the proposed model of turbulence in stably stratified media including the notion of progressive extraction of kinetic energy from the turbulence and the resultant vast reduction in the dissipation rate $\epsilon$.

The author expresses his gratitude to W. E. Gordon for many helpful discussions and suggestions during the conduct of this investigation; and to the Lincoln Laboratory, Massachusetts Institute of Technology, for so kindly inviting the author to visit its Tropospheric Propagation Group in order that he might analyze the results of certain of its radio experiments.

\section{References}

[1] H. G. Booker and W. E. Gordon, Theory of radio scattering in the troposphere, Proc. Radio Wave Propogation Symp., Navy Electronics Lab., San Diego, Calif. (1949); also Proc. IRE 38, 401 (1950).

[2] E. C. S. Megaw, Scattering of electromagnetic waves by atmospheric turbulence, Nature 166, 1100 (1950).

[3] F. Villars and V. F. Weisskopf, The scattering of electromagnetic waves by turbulent atmospheric fluctuations, Phys. Rev. 94, 232 (1954).

[4] F. Villars and V. F. Weisskopf, Scattering of radio waves by turbulent fluctuations of the atmosphere, Proc. IRE 43, 1232 (1955).

[5] K. A. Norton, P. L. Rice, and L. E. Vogler, Use of angular distance in estimating transmission loss, Proc. IRE 43, 1488 (1955)

[6] R. A. Silverman, Turbulent mixing theory applied to radio scattering, J. Appl. Phys. 27, 699 (1956).

[7] H. T. Friis, A. B. Crawford, and D. C. Hogg, A reflection theory for propagation beyond the horizon, Can. Conv. IRE, Toronto, Canada (1956); Bell System Tech. J. 36, 627 (1957); also A. B. Crawford, D. C. Hogg, and W. H. Kummer, Bell System Tech. J. 38, 1067 (1959), for a further discussion of the variability of wavelength dependence as influenced by laver size relative to a subtended Fresnel zone. (Ed.)

[8] K. A. Norton, Point-to-point radio relaying via the scatter mode of tropospheric propagation, IRE Trans. CS 4, 39 (1956); unpublished report (1955), in particular the Note added July 28, 1956, introducing the lognormal modification.

[9] R. B. Muchmore, private communication to K. A. Norton (1955), reference [8].

[10] R. Bolgiano, Spectrum of turbulent mixing, Phys. Rev. 108, 1348 (1957).

111] A. D. Wheelon, On the spectrum of a passive scalar mixed by turbulence, J. Geophys. Research 63, 849 (1958).

[12] R. Bolgiano, Jr., On the role of convective transfer in turbulent mixing, J. Geophys. Research 63, 851 (1958),

[13] G. K. Batchelor, The scattering of radio waves in the atmosphere by turbulent fluctuations in refractive index (Cornell Univ., School of Elec. Eng. Research Rept. EE 262, Ithaca, N.Y., 1955).

[14] R. Bolgiano, The role of turbulent mixing in scatter propagation, IRE PGAP Trans. AP-6, 161 (1958).

[15] J. R. Bauer, The suggested role of stratified elevated layers in transhorizon short-wave radio propagation (Lincoln Lab., Tech. Rept. No. 124, Bedford, Mass., 1956).

[16] J. H. Chisholm, J. F. Roche, and W. J. Jones, Experimental investigations of angular scattering and multipath delays, Proc. of Combined Tech. Session, URSIIRE, Washington, D.C. (1957).

[17] R. Bolgiano, Jr., Wavelength dependence in transhorizon, propagation, Proc. IRE 47, 331 (1959).

[18] G. K. Batchelor, The theory of homogeneous turbulence (Cambridge Univ. Press, London, England, 1953).

[19] H. Charnock, Note on eddy diffusion in the atmosphere between one and two kilometers, Quart. J. Roy. Meteorol. Soc. $\boldsymbol{7}$ \%, 654 (1951).

[20] R. Bolgiano, Jr., Turbulent spectra in a stably stratified atmosphere, J. Geophys. Research 64, 2226 (1959).

[21] G. K. Batchelor, Small-scale variation of convected quantities like temperature in turbulent fluid, pt. 1: General discussion and the case of small conductivity, J. Fluid Mech. 5, 113 (1959).

[22] G. K. Batchelor, I. D. Howells, and A. A. Townsend, Small-scale variation of convected quantities like temperature in turbulent fluid, pt. 2: The case of large conductivity, J. Fluid Mech. 5, 134 (1959).

[23] F. Gifford, Jr., Relative atmospheric diffusion of smoke puffs. J. Meteorol. 14, 410 (1957).

[24] R. Bolgiano, Jr., A meteorological interpretation of wavelength dependence in transhorizon propagation (Cornell University, School of Elec. Eng. Research Rept. EE 385, Ithaca, N.Y., 1958).

Boulder, Colo.,

(Paper 64D3-52) 